\title{
A novel method to investigate bladder wall behavior by acceleration and pressure sensing
}

\author{
Tristan Weydts ${ }^{a *}$, Luigi Brancato ${ }^{a}$, Mohammad A. Soebadi ${ }^{b}$, Dirk De Ridder ${ }^{b}$ \\ and Robert Puers ${ }^{\text {a }}$ \\ a KU Leuven, Dept. of Electrical Engineering, MICAS, Kasteelpark Arenberg 10, 3001 Leuven, Belgium \\ b KU Leuven, Laboratory of Experimental Urology, Herestraat 49, 3000 Leuven, Belgium \\ * Correspondence: tristan.weydts@kuleuven.be; Tel.: +32-16-328082 \\ Full postal address: Kasteelpark Arenberg 10 bus 2440, 3001 LEUVEN, Belgium
}

\section{Abbreviations $^{1}$}

\section{Abstract}

Bladder dysfunctions form a continuously growing problem as the ageing population in our society is steadily increasing. However, scientific knowledge about the fundamental principles underlying bladder diseases is still lacking. Conventional cystometry-based methods for assessing bladder function are quite limited in their scope. In this paper, a novel device is introduced to research the bladder. An exploratory device was designed for measuring bladder wall contractions in rats using accelerometers. Later, a fully implantable system was created to extend the measurements to larger animal models. The batterypowered implant provides a wireless readout of multiple sensors with synchronous visualization. An intelligent algorithm is used to optimize the power consumption and to extend the autonomy after implantation, yielding a useable measurement time of 16 continuous days and several months of standby-time.

\section{Keywords}

implantable systems; bio-telemetry; bladder monitoring; sensor networks

\section{Introduction}

The number of people suffering from bladder dysfunctions is estimated to be well into the hundreds of millions worldwide [1]. Diseases affecting the sensation of urgency include overactive bladder syndrome, urinary incontinence and lower urinary tract symptoms, the exact functionalities of which are still poorly understood by modern medicine.

Traditional methods of assessing bladder function are based on intraluminal pressure measurements through a catheter, urine flow and volume measurements, and in some cases electromyography (EMG) of the sphincter muscle [2,3]. These methods are quite limited in their scope, as they provide only a summation of whole bladder activity and no localized bladder information. As such, they are mostly

\footnotetext{
${ }^{1}$ EMG, electromyography; PCB, printed circuit board; USB, universal serial bus; I2C, inter-integrated circuit; IPHFO, intraluminal pressure high frequency oscillations; UART, universal asynchronous receiver-transmitter; $P_{\text {ves, }}$ vesical pressure; $\mathrm{P}_{\text {det }}$, detrusor pressure
} 
useful as a diagnostic tool rather than for providing new medical insights into the bladder. Moreover, the catheter and EMG leads, which are connected to an external measurement system, are a discomfort to the patient and also limit measurement duration to no more than several voids.

The hypothesis of autonomous bladder function, described in [4,5], suggests the bladder senses its volume and pressure, which is linked to the patient's feeling of urgency, by using localized contractions. Those theorized bladder wall movements might occur prior to voiding, as well as during voiding. Moreover, these local contractions may as well occur at random time intervals, unrelated to the emptying of the bladder. In fact, the autonomous function of the bladder is increased in absence of central nervous system efferent inhibition. This insight is promising for patients that experienced spinal trauma and suffer from urinary incontinence.

To obtain a better understanding of the fundamental bladder mechanics, a new method for assessing bladder functionality is explored in this paper. In a first study, an exploratory device for acute in vivo measurement of acceleration of the bladder wall of rats is proposed. The acceleration results are compared to conventional catheter pressure measurements and the design of the system, along with a measurement during voiding, is discussed in Section 2. Using the findings from the first phase, a fully implantable system is developed which monitors bladder wall accelerations and pressure and is described in Section 3. The implant has sensors placed in several localized spots, to compare localized bladder wall movements in different positions of the bladder. The system is fully implantable for several months while being unobtrusive for the patient. This novel approach will provide physicians with valuable new information about the inner operation of the bladder, the conditions that cause bladder dysfunctions and the possible relation between bladder muscle contractions and the sensation of urgency.

\section{Exploratory study of physiological measurements of the bladder}

This section discusses the methodology used to facilitate exploratory investigations of physiological functionality of the bladder: the placement of an accelerometer on the bladder of a rat.

\subsection{Description of the device}

In order to perform in vivo measurements of bladder functionality, a selection of sensors needs to be made according to the following specifications listed. The selected devices must inherently be biocompatible or at least permit a biocompatible packaging. The physical dimensions of the sensors need to be as small as possible, preferably smaller than $5 \times 5 \times 2 \mathrm{~mm}$. The power consumption during measurement as well as in idle state should be minimized to allow a long operating time of the battery (at least several days of autonomy). The sampling frequency is at least twice the bandwidth of the measured signal. However, given the novelty of these measurements, the bladder signals and their bandwidth are unknown. Therefore, inspiration to set the specifications needs to be sought with previous experience, signals from other medical fields and expert medical opinion. As shown in a smooth muscle experiment [6], a good estimate for the highest frequency components contributing to 
smooth muscle movements is in the order of $50-100 \mathrm{~Hz}$. Therefore, the assumption is made that the bladder does not exhibit movements with frequency components more than $100 \mathrm{~Hz}$. The range of inertial measurements from bladder contractions is expected not to exceed $\pm 2 \mathrm{~g}$. The resolution required is unknown and should thus be as high as possible. The pressure difference of the bladder between its empty and filled state, as measured with intraluminal catheters, is 300 mbar. A resolution of $0.1 \mathrm{mbar}$ is sufficient to measure any pressure variations in classical cystometry. Although the use of an EMG system has been shown to provide interesting results concerning bladder contractions [7] and can be made implantable, , the choice was made to limit the system to inertial and pressure measurements in order to avoid biocompatibility as well as noise problems.

A miniature 3-axis accelerometer (Bosch Sensortec BMA280) is mounted at the end of a $25 \mathrm{~cm}$ long, flexible PCB. Besides this accelerometer, the optional functionality was added to monitor bladder pressure, so a digital pressure sensor (TE Connectivity MS5637) was added close to the accelerometer. The sensors are selected for their compact size and low power consumption. The accelerometer measures $2 \times 2 \times 0.95 \mathrm{~mm}$ and consumes $130 \mu \mathrm{A}$ in active mode. It is configured with range of $\pm 2 \mathrm{~g}$, resolution of 14 bits and sampled at $500 \mathrm{~Hz}$. The pressure sensor has dimensions of $3 \times 3 \times 0.9 \mathrm{~mm}$ and an average current consumption of $150 \mu \mathrm{A}$ during sampling. The pressure signal is sampled at the same frequency of $500 \mathrm{~Hz}$, has a range of 0-2 bar and 24-bit resolution. The assembled device is depicted in Figure 1.

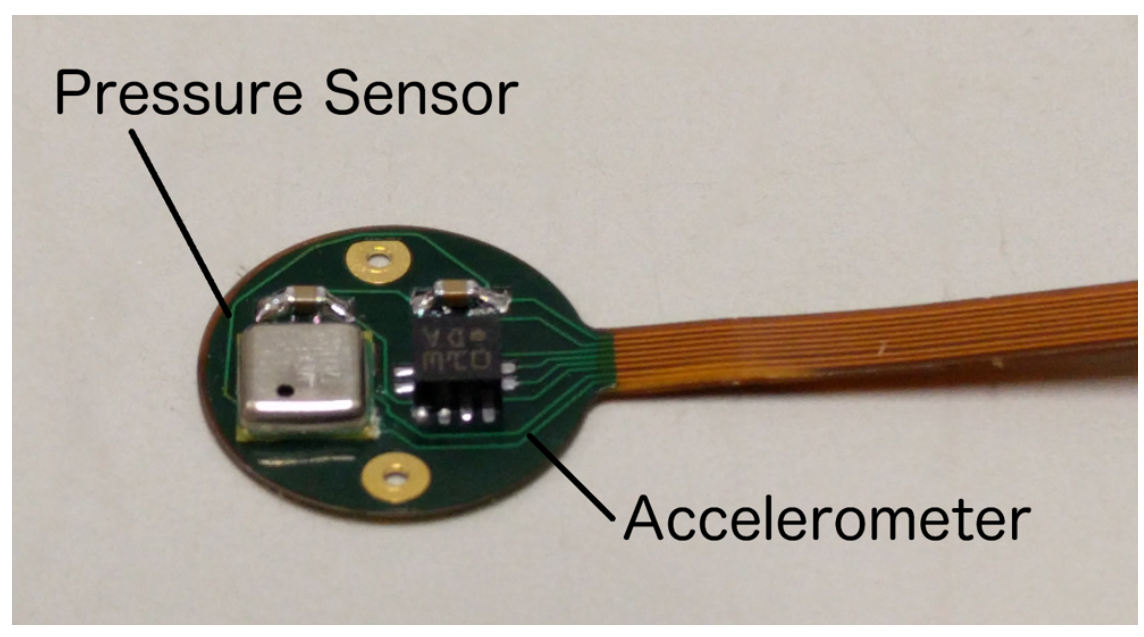

Fig. 1. The bladder wall measurement sensors, an accelerometer and pressure sensor mounted on a flexible PCB.

A biocompatible packaging is necessary to protect the sensors from the body fluids, as well as to shield the organs from the electronics. The MS5637 is a barometric pressure sensor designed for operation in air and is covered by a metal cap to protect the electronics. However, since a mechanical coupling is needed between the pressure sensitive membrane under the protective cover and the bladder wall, the metal cap must be removed, exposing two silicon dies and the bondwires connecting 
them. Three packaging layers are used: (1) parylene-C with a thickness of $5 \mu \mathrm{m}$ is applied by chemical vapor deposition and provides low water permeability, (2) a medical grade epoxy is used to mechanically reinforce the electrical connections of the sensors, as well as the dies and bondwires of the pressure sensor, taking special care not to cover the sensor membrane, and ( 3 ) the device is dip-coated in medical grade PDMS. The factory calibration of the pressure sensor is invalidated by the application of the packaging layers, so it needs to be recalibrated. The BMA280 acceleration sensor, however, is completely sealed and unaffected by the packaging, so the factory calibration and baseline drift correction remain valid.

The flexible PCB is connected to a readout board that contains a microcontroller. The digital samples of the sensors are read out over a synchronous serial I2C interface and transmitted to a personal computer over USB. The readout board and acquisition software can simultaneously measure and plot two accelerometers and two pressure sensors.

\subsection{Implantation procedure and measurement}

All experimental procedures are performed in accordance with applicable institutional and national guidelines. The accelerometer is implanted in a male Sprague-Dawley rat. Under urethane anesthesia $(1.3 \mathrm{~g} / \mathrm{kg}$ s.c.), the bladder is exposed by laparotomy. Continuous saline infusion cystometry at 100 $\mu \mathrm{L} / \mathrm{min}$ is performed through a flared-tip suprapubic catheter (PE-50, Intramedic) with luminal pressure registration (BIOPAC MP150). The catheter perforates the outer bladder muscle, called detrusor. The small size of the bladder prevents the use of the pressure sensor, so only the accelerometer is used. To prevent the accelerometer to move away from its position on the bladder muscle and to ensure an intimate connection of the sensor to the bladder wall, the tip of the PCB is clamped under a patch of abdominal tissue using stitches. The implanted device is depicted in Figure 2.

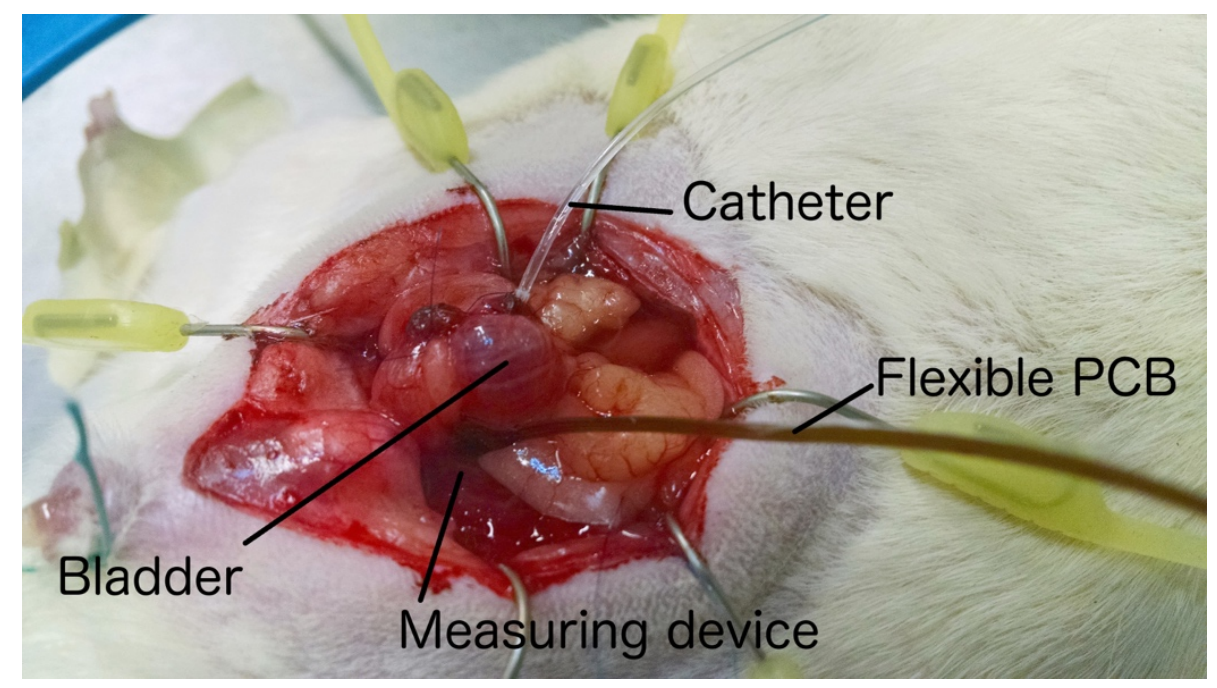

Fig. 2. Placement of an accelerometer on the bladder of a male rat, covered by abdominal tissue. A pressure catheter, used for filling the bladder, perforates the detrusor. 
A continuous measurement is carried out during the entire infusion, resulting in records of several voiding events. The conventional rise in pressure at the onset of each voiding event was clearly observed, as well as the aftercontraction. During the voiding itself, intraluminal pressure high frequency oscillations (IPHFO) are visible in the pressure signal, as described in [8]. The IPHFO, traditionally only described in the pressure signal, have a clear counterpart in the acceleration signal, as shown in Figure 3. The acceleration signal shown is the one corresponding to the z-axis which is located perpendicular to the accelerometer chip and the bladder wall. The raw signal is bandpass-filtered between 2 and $100 \mathrm{~Hz}$ to remove the gravitational component. A zoomed view of the signal during the IPHFO is shown as well. We postulate that accelerometers can serve a beneficial role as an additional tool, extending traditional urodynamic methods with a new sensor that has a high sensitivity for bladder wall movement detection.
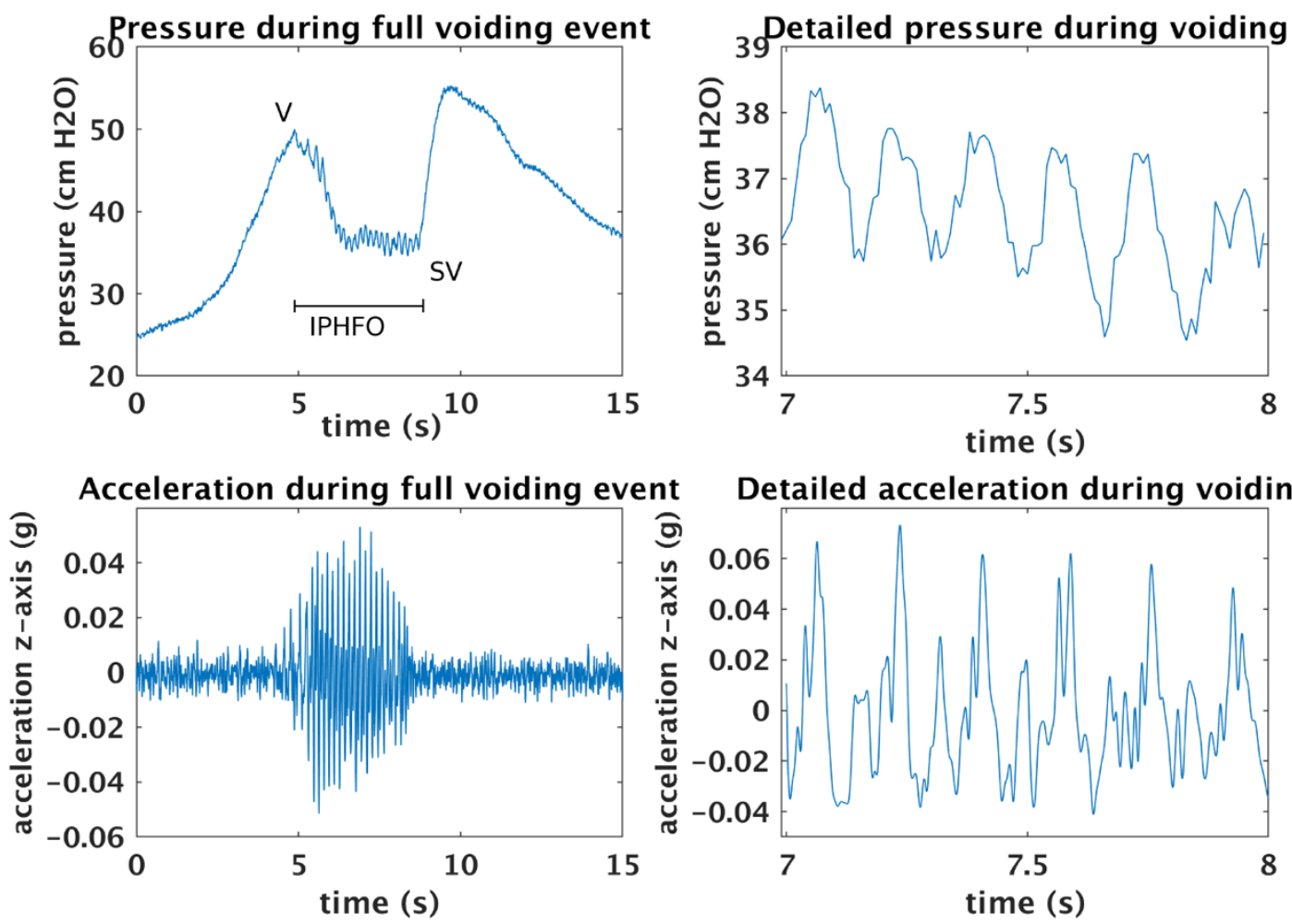

Fig. 3. Pressure and $z$-axis acceleration during a voiding event of a rat. $V$ denotes the onset of the voiding and SV denotes the end. A full voiding cycle is shown, along with a detailed view of pressure and acceleration during the IPHFO.

The obtained results of this exploratory device, while limited in scope, reveal a promising capability of accelerometers as a new type of sensor, which merits further exploration. A new, larger animal model is examined that more closely matches human anatomy. Göttingen minipigs are often used in clinical studies for their anatomical resemblance to humans and ease of handling [9-11]. However, they do no void under anesthesia, which requires a system that allows to measure voiding while the animals are 
awake. For this purpose, a chronic measurement system is designed for long-term monitoring of localized bladder contractions and is described in the next section.

\section{A fully implantable bladder device: submucosal accelerations of the bladder wall}

3.1. System design

The long-term implantable bladder device is an attempt to extend the previous measurements to larger animal models, for longer periods of time and while the animals are awake and unaffected by the measurements. The goal is to implant multiple accelerometers to characterize localized bladder contractions, as well as measure the bladder pressure without need of a catheter, as was done in the previous experiment. The sensors are placed between the detrusor muscle, which forms the outer layer of the bladder, and the mucous membrane (mucosa) on the inside of the bladder. This configuration is referred to as a submucosal implantation and has the benefit of keeping the sensors in place. Moreover, as shown by [12], submucosal pressure measurements show a good correlation with the subluminal pressure of the bladder, provided proper calibration of the sensor is performed. The implant consists of two independent subsystems that will be explained in the following sections: the sensor system, which houses all sensors and is placed on the bladder, and the telemetry and power system, which is placed subcutaneously. A block diagram of the complete system is shown in Figure 4. 


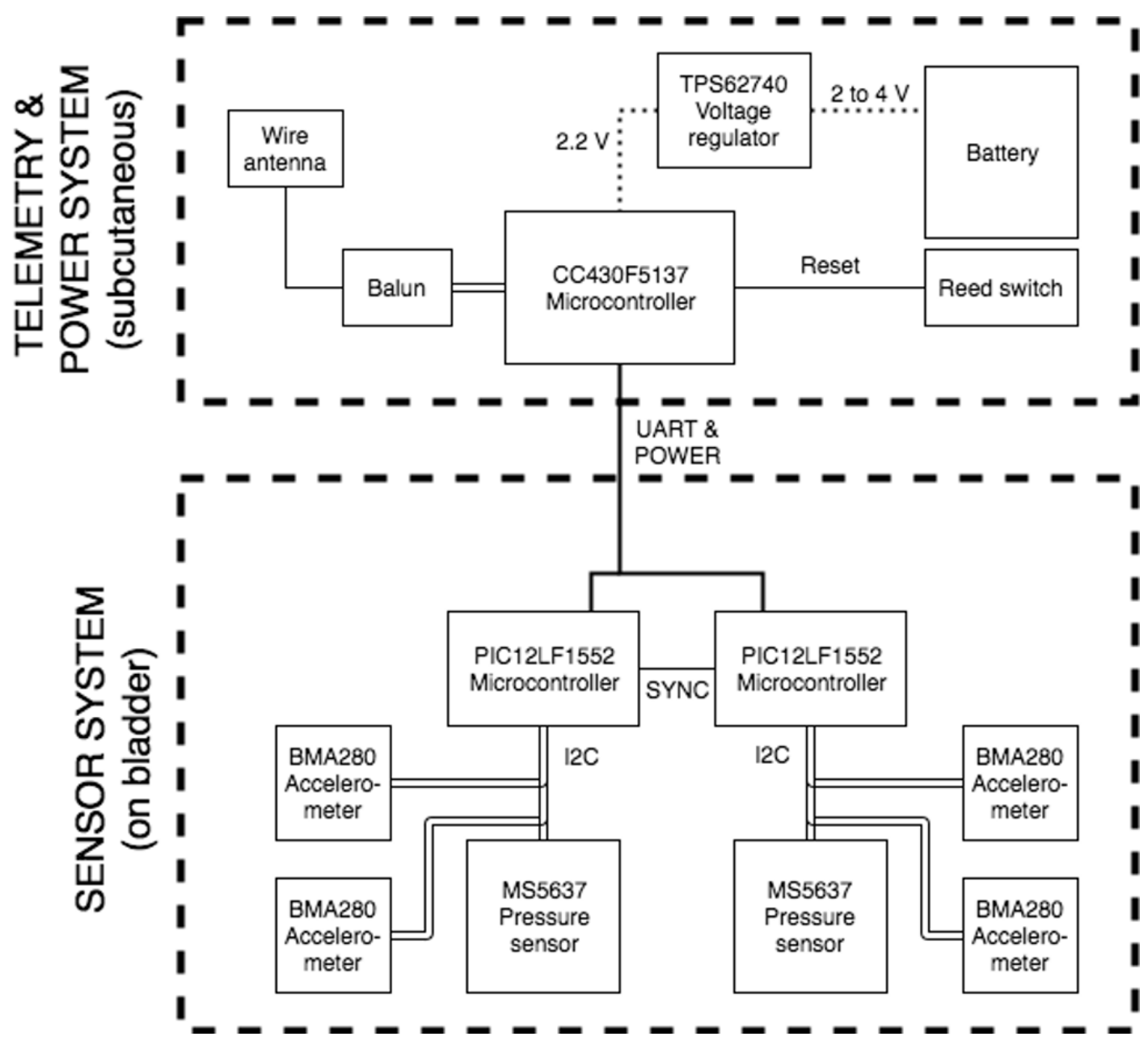

Fig. 4. Block diagram of the system, consisting of two subsystems.

\subsubsection{The sensor system}

The bladder is an organ that drastically changes size between its full and empty state; the difference in volume can reach up to $100 \%$ [13]. It is thus important for the measurement system, which wraps around the bladder, to be able to move and stretch along with the bladder and at the same time avoid any physical stress on the muscle. The flexibility and stretchability of the sensor system is an important aspect of the physical design. In contrast, the sensors must be located at fixed positons and shall not move with respect to the bladder muscle, which requires flexible and stretchable interconnects. The sensor system is made on a flexible polyimide substrate that has a wavy shape to allow for some stretchability. The resulting shape has a central island placed on the bladder, which connects to three distal ends housing the sensors and implanted submucosally. Figure $5 a$ and $5 b$ show the intended location on the bladder and the fabricated sensor system respectively. 


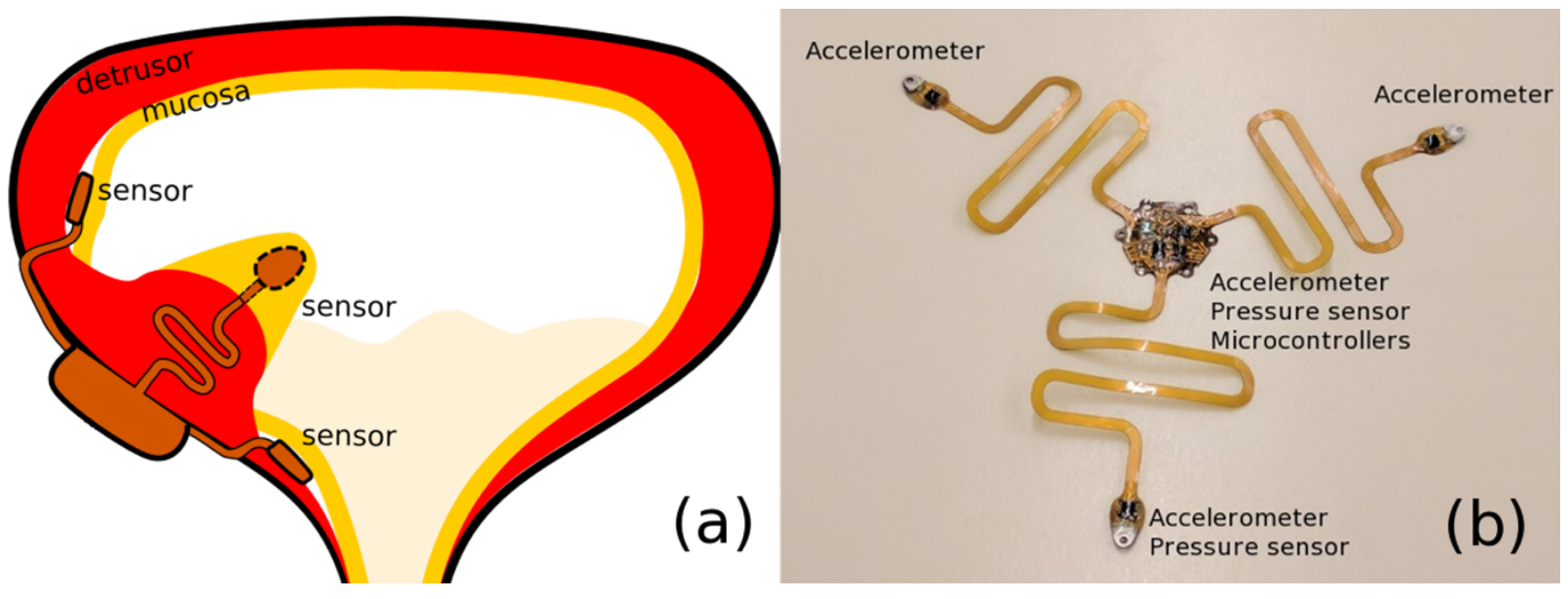

Fig. 5. (a) The placement of the sensor system around the bladder. (b) The fabricated sensor system, which is built on a flexible substrate that shows some stretchability.

Bladder wall movement detection is done at four different locations, by four accelerometers (Bosh Sensortec BMA280). The sensors are placed at each end of the three arms of the structure and one accelerometer on the central island. As in the previous section, the system is also equipped with two absolute pressure sensors (TE Connectivity MS5637), one which is implanted submucosally behind one of the accelerometers and thus facing the internal bladder volume, and one which measures abdominal reference pressure, outside of the bladder on the central island. All six sensors are read out over an I2C digital interface bus. The sample frequency of all sensors is the same and can be chosen to be 50, 125 or $250 \mathrm{~Hz}$. There are two 8-bit microcontrollers (Microchip PIC12LF1552) on the central island, each connected to two accelerometers and one pressure sensor. The reason is twofold: firstly, the BMA280 accelerometer sensors have only two possible 12C addresses and the MS5637 pressure sensor has an address that cannot be altered. Therefore, it is not possible to connect all sensors to the same bus. Secondly, the parallelization allows sensor data from 3 sensors to be read out simultaneously with the transmission of data of 3 other sensors to the telemetry system. Thus, in order maximize throughput of the system, the microcontrollers take turns sampling sensors and writing the resulting data to the telemetry subsystem over a shared UART connection. To guarantee correct timings, both microcontrollers indicate their state using a synchronization line. The microcontrollers are the first line of defense in case there is a defective sensor; rather than blocking the entire $12 \mathrm{C}$ bus, the error can be detected and future readings of the sensor can be skipped. This makes the sensor system quite independent and error-resilient.

\subsubsection{The telemetry and power system}

The implant is used as a diagnostic tool for physicians. As such, it is desirable to have a real-time readout and visualization of the measurement data without the need for a flash memory. Since the system is hermetically sealed and implanted, a fully wireless link is required. The battery, electronics used to power the implant and the wireless telemetry system are placed on a separate PCB and implanted subcutaneously, several centimeters under the skin and fat layers. 


\subsubsection{Telemetry}

For wireless transmission, the $433-435 \mathrm{MHz}$ ISM band is a commonly used and freely available RF channel. A fully integrated transceiver is desirable for a compact implantable device. Therefore, the Texas Instruments CC430 is used, which is a 16-bit microcontroller with integrated CC1101 RF core. The CC430 chip measures $7 \times 7 \times 1 \mathrm{~mm}$ and enables sub 1GHz communication with only an external balun and antenna required. The microcontroller functions as the brain of the implant, managing the sampling and sleep state of the sensors, as well as the wireless communication protocol.

There are many possible antenna designs for communication at $430 \mathrm{MHz}$, including wire antennas, patch antennas, PCB antennas and custom designed 3D antennas [14-16]. The difficulty in implantable systems is getting the electromagnetic waves outside of the body. Because the antenna is surrounded by biological tissue with relative electrical permittivity $\varepsilon_{r}$ ranging from 5 to 50 [17], the antenna will be de-tuned. Since the variation in $\varepsilon_{r}$ is high, it is desirable to choose an antenna with a high bandwidth. A monopolar wire antenna was chosen with length of a quarter-wavelength, which is $17.5 \mathrm{~cm}$. Although other antenna designs are much more compact, the simplicity of a wire antenna is a clear design advantage. The penetration depth at $400 \mathrm{MHz}$ is $5 \mathrm{~cm}$ in muscle and $30 \mathrm{~cm}$ in fat tissue [18], but since the telemetry system is placed under the skin, a fully optimized antenna is less important.

The telemetry system has two tasks: to transmit the measured data and to enable bidirectional communication with a fixed base station. The CC430 transceiver uses a packet-based protocol that adds the necessary bytes for identification, addressing and error detection, which allows it to use interruptbased routines to control the communication flow. The implant application software uses the implemented low-level functionality to create a smart communication scheme that minimizes the power consumption of the implanted device. The wireless communication is used to configure settings of the implant, such as the frequency of communication, transmitting power, several configurable timers, as well as starting the stream of sensor data. The communication system can be in one of three states: the idle state, the transmitting state or the receiving state.

\subsubsection{Power management}

The power consumption of the battery-operated implant is critical for the autonomy of the system, if the measurement system is to be used for longer durations. Moreover, a method to reduce battery drain in the period between packaging and the final implantation, is strongly desired.

The implant is powered by a small form factor, high capacity battery. A thin-cell CP502440 primary lithium manganese dioxide battery is used, which measures $24 \mathrm{~mm} \times 40 \mathrm{~mm} \times 5 \mathrm{~mm}$, with a capacity of $1200 \mathrm{mAh}$, a nominal voltage of $3 \mathrm{~V}$ and a cut-off voltage of $2 \mathrm{~V}$. The voltage of the battery is downconverted to $2.2 \mathrm{~V}$ by a buck-converter (Texas Instruments TPS62740) to provide a stable supply voltage over $95 \%$ of the capacity of the battery. The TPS62740 also has an integrated load switch, which is used to power the sensor subsystem so that it can be completely switched off when it is not in use, reducing the current consumption during idle operation. 
Power measurements reveal that the current consumption in the receiving state is $13 \mathrm{~mA}$. Since this is 1300 times higher than the idle state $(10 \mu \mathrm{A})$, the time spent in receiving state must be minimized. The default state of the implanted device is idle state, during which it will periodically transit to the receiving state for 1 millisecond, which is long enough to transmit any pending commands. The power consumption of the base station is not important since it is powered through USB, so its default state is the receiving state so that it can always detect communication from the implant.

The power system includes a reed switch, connected to the reset pin of the CC430 microcontroller. The reed switch can be triggered by a magnet, in vitro as well as in vivo, to bring the implant to a known state if it becomes unresponsive, force the implant to switch to the receiving state, or activate the system after it has been placed in a deep sleep mode to conserve power.

Table 1 reveals measurements for the power consumption of the different modes of the implant. As mentioned before, residing in RF receive mode is costly in terms of power but necessary for communication. To reduce the average power consumption, a compromise is made between responsiveness and battery life. The implant stays in the receiving state for one millisecond every second, and the resulting the duty cycle of $1 / 1000$ yields an average power consumption of $0.023 \mathrm{~mA}$ with a guaranteed opportunity of communication every second, practically extending battery life by 1000 times. As depicted in Table 1, the described settings result in a theoretical autonomy of the system of 375 hours of continuous transmitting, 2000 days hours of periodic wakeup, or any combination of both. Since the periodic wakeup state consumes negligible current, the battery life is limited only by the transmit state and is around 5 to 15 days of continuous measurement, depending on the chosen sample rate.

Table 1. Power consumption of device subsystems during the different states.

\begin{tabular}{|c|c|c|c|c|}
\hline \multicolumn{2}{|l|}{ State } & $\begin{array}{l}\text { Current } \\
\text { consumption } \\
\text { sensor system }\end{array}$ & $\begin{array}{l}\text { Current } \\
\text { consumption } \\
\text { telemetry }\end{array}$ & $\begin{array}{l}\text { Battery life in this } \\
\text { state (with capacity } \\
\text { of } 1200 \mathrm{mAh} \text { ) }\end{array}$ \\
\hline \multicolumn{2}{|l|}{ Receive state } & - & $13 \mathrm{~mA}$ & $90 \mathrm{hr}$ \\
\hline \multicolumn{2}{|l|}{ Idle state } & - & $0.01 \mathrm{~mA}$ & 5000 days \\
\hline $\begin{array}{l}\text { Data stream: } \\
\text { sampling and } \\
\text { transmit state }\end{array}$ & $\begin{array}{l}50 \mathrm{~Hz} \\
125 \mathrm{~Hz} \\
250 \mathrm{~Hz}\end{array}$ & $\begin{array}{l}1.1 \mathrm{~mA} \\
2.5 \mathrm{~mA} \\
5.0 \mathrm{~mA}\end{array}$ & $\begin{array}{l}2.1 \mathrm{~mA} \\
3.4 \mathrm{~mA} \\
6.2 \mathrm{~mA}\end{array}$ & $\begin{array}{l}375 \mathrm{hr} \\
200 \mathrm{hrs} \\
107 \mathrm{hrs}\end{array}$ \\
\hline \multicolumn{2}{|c|}{$\begin{array}{l}\text { Periodic wakeup state } \\
\text { (1 ms receive, } 999 \mathrm{~ms} \\
\text { idle) }\end{array}$} & - & $0.023 \mathrm{~mA}$ & 2000 days \\
\hline
\end{tabular}

The telemetry and power system is connected to the sensor system via a stretchable interconnect described in [19], as shown in Figure 6. 


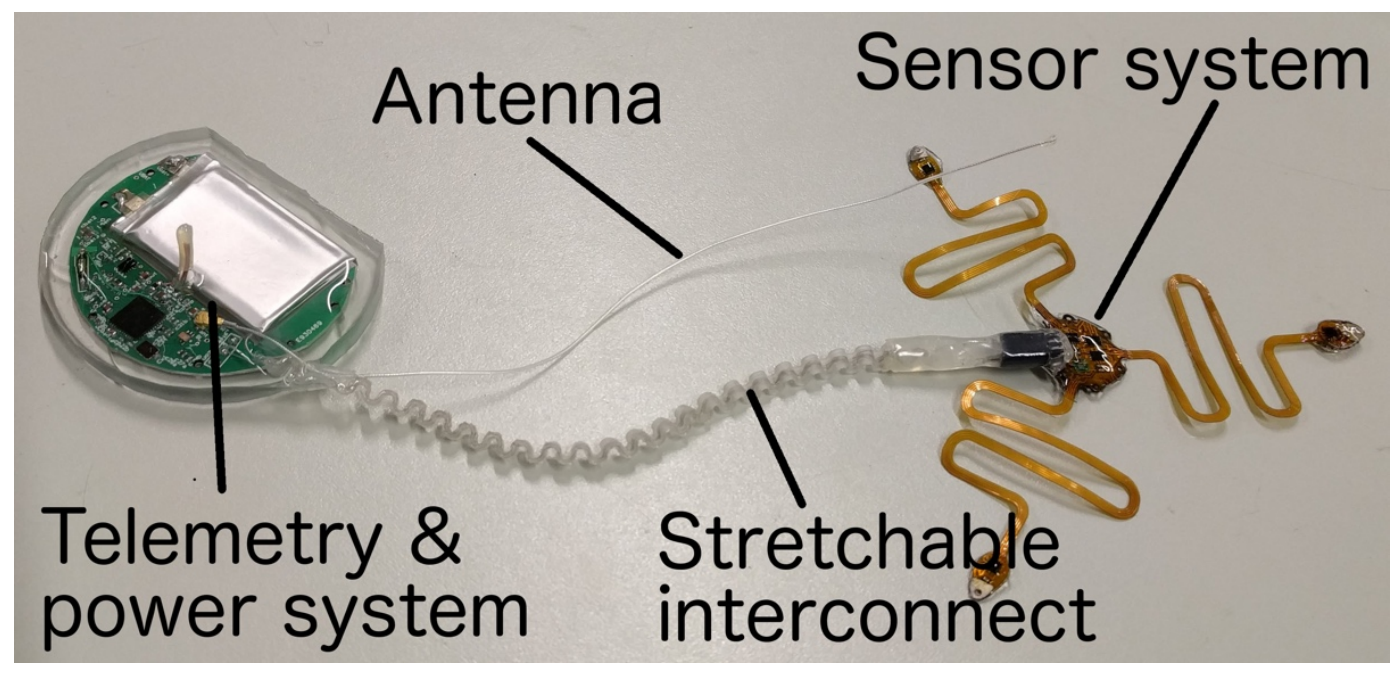

Fig. 6. The assembled implant: the sensor system connected to the telemetry and power system through a stretchable interconnect.

\subsection{Functional cadaver tests of the implant}

After extensive in vitro tests to confirm the functionality and performance of the implant, a series of cadaver tests were performed. Firstly, to verify the feasibility of implanting a pressure sensor and accelerometer under the detrusor muscle without perforating the mucosa and causing a leak in the bladder, a functional cadaver test is performed. The shape and stretchability of the sensor subsystem is also tested. After filling the bladder of a porcine cadaver, the sensor system is placed to match its contours. Submucosal implantation is performed by making a superficial incision into the inflated detrusor muscle, placing the sensor islands on the exposed mucosa and stitching the detrusor over the device. After abdominal closure. measurements from the sensors are continuously taken and transmitted over USB to a personal computer for visualizing and storing the data from four accelerometers, one submucosal and one abdominal pressure sensor. Figure 7 shows the implant located on the bladder.

Secondly, the required transmission power for continuous telemetry was tested by implanting the telemetry and power system subcutaneously in a fresh sheep cadaver. A transceiver placed under the skin with transmission power of $10 \mathrm{dBm}$ measured a byte-error rate of less than $0.1 \%$ at a distance of 2 $\mathrm{m}$. 


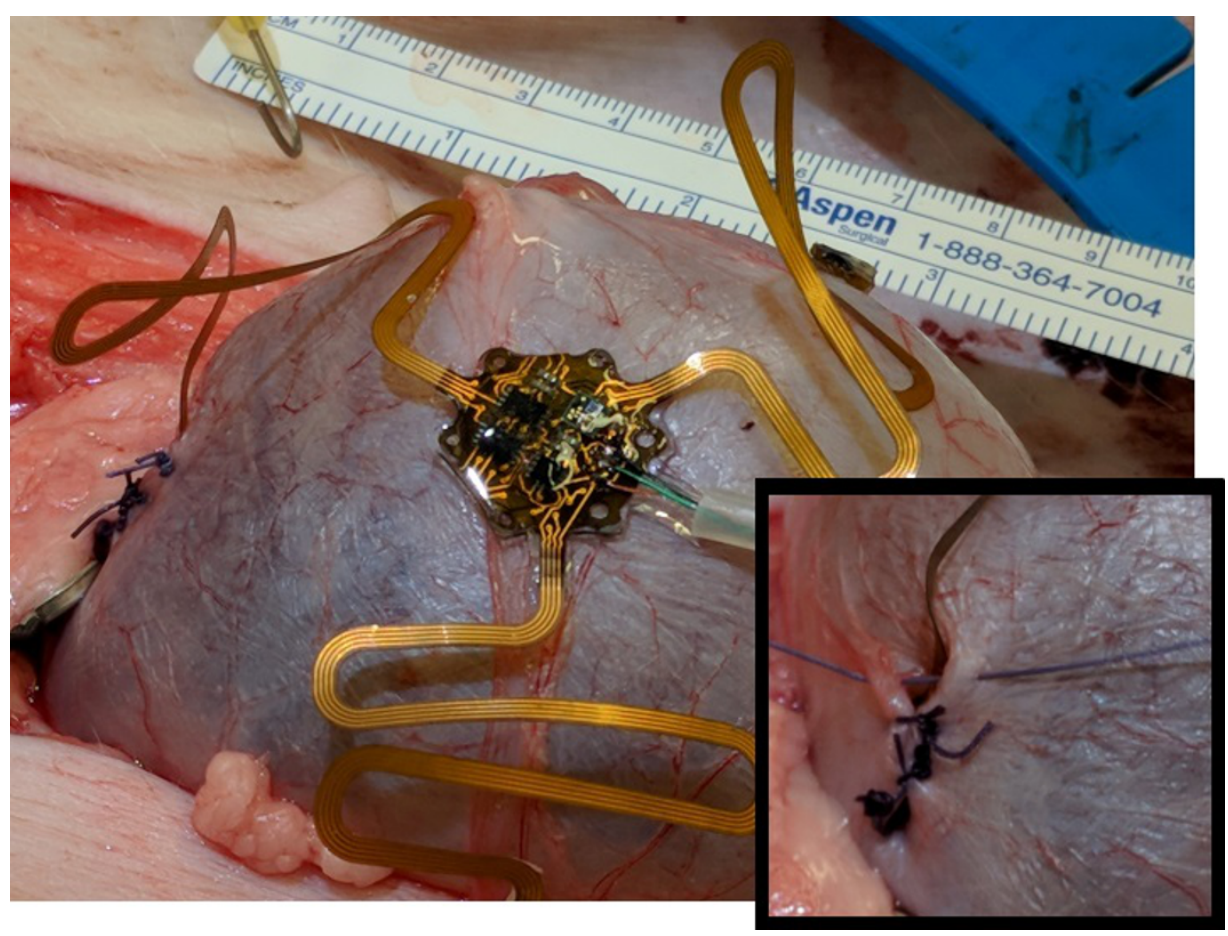

Fig. 7. Implanted bladder sensor system on a pig cadaver prior to abdomen closure. One accelerometer and the submucosal pressure sensor are placed deep in the abdomen, on the bladder apex. Two other accelerometers are placed on the left and right side of the bladder. A closeup of the submucosal implantation is shown as well.

We aim to demonstrate that the submucosal pressure signal, which is referred to as detrusor pressure $P_{\text {det }}$, shows a good agreement with the actual vesical pressure inside the bladder $P_{\text {ves, }}$ measured through a catheter by an air-charged catheter. Some functional measurements of the sensors are depicted in Figure 8, where a series of six manual injections through a syringe were performed to inflate the bladder while measuring the pressure signal. The detrusor pressure sensor was calibrated in air at $20.0^{\circ} \mathrm{C}$ one week before the test. During this week, the offset calibration parameter was changed by the relaxation of the packaging materials and sensor membrane. However, the sensitivity of the membrane remained the same. Thus, a new baseline pressure was measured in the operating room before implantation to compensate for the change in offset. The relative pressure signals were calculated using the new offset, resulting in the graph in Figure 8. $P_{\text {det }}$ and $P_{\text {ves }}$ clearly show strong similarities, although the amplitude of the detrusor pressure signal is lower. A difference between both pressure measurements is expected, since the physiological signals are different and the effect of the mucosa on the pressure membrane is unknown. However, the measurement is also influenced by the temperature sensitivity of the sensor, the difference in cadaver temperature and infused saline solution, and possibly the presence of an air bubble in the bladder. During in vivo tests, temperature drift and air bubbles in the bladder would not be issues, since the temperature is very stable and the bladder does not contain air. An in vivo calibration could be made after implantation to get more accurate estimates of $\mathrm{P}_{\text {ves. }}$. The acceleration signals were also measured for the sake of validating the functionality of the sensor system. However, due to the cadaveric nature of the test, no meaningful physiological data was measured, and 
the measured, unfiltered, accelerations are only caused by the handling of the animal and the gravitational acceleration. Nevertheless, full operation of the multisensory system could be proven.
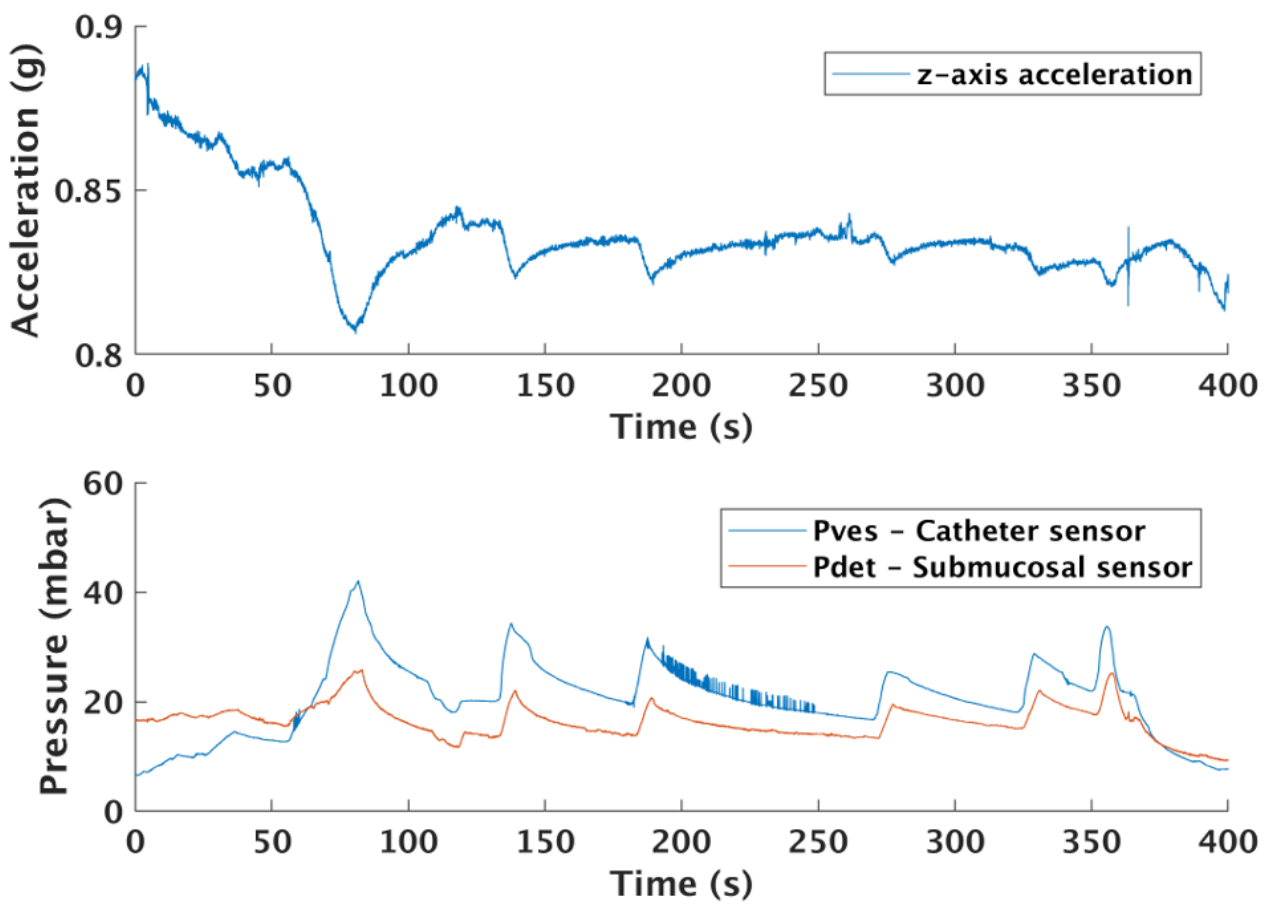

Fig. 8. Acceleration, intraluminal catheter pressure and submucosal pressure during manual inflation of the bladder of a pig cadaver. All pressures are relative to atmospheric pressure.

\subsection{In vivo measurements of the voiding bladder}

After the functional cadaver tests, the system was implanted in a live Göttingen minipig. The bladder was filled with saline solution by means of a pump through a catheter and voiding events were recorded. Figure 9 depicts some preliminary signals measured during one voiding event. They clearly show that the submucosal pressure sensor is sensitive to a local pressure signal in the bladder during voiding.. The accelerometer signal exhibits small vibrational signals before and during the voiding event, which prove the feasibility of this device and will be further explored after more in-vivo tests are done. At present extensive measurements are being performed, which will form the basis of next research. 

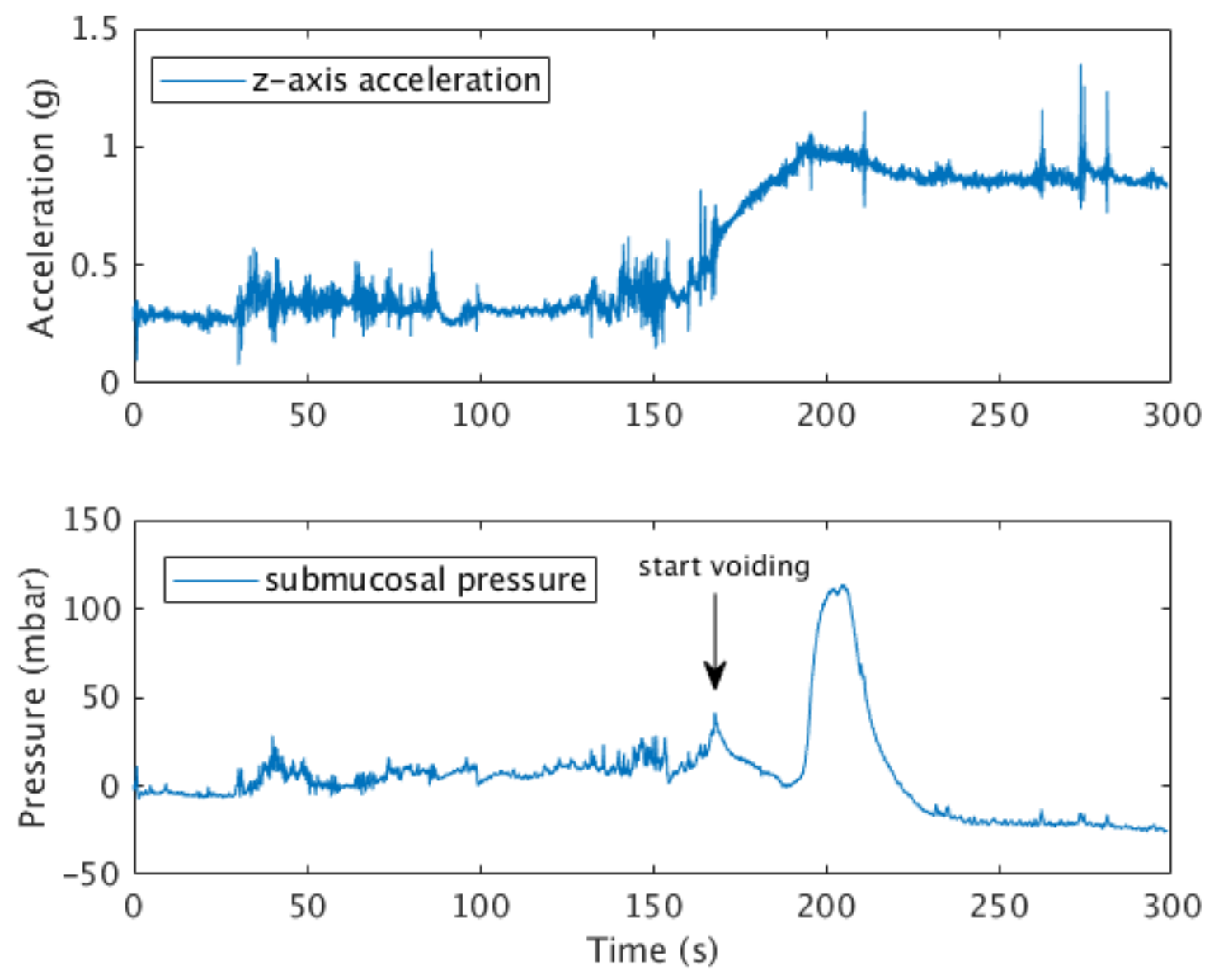

Fig. 9. An acceleration signal, alongside submucosal pressure during the voiding of the bladder of a pig. The pressure signal is relative to the baseline pressure measured between voiding events.

\section{Conclusions}

In order gain better understanding of physiological behavior of the urinary bladder, a novel method for assessing bladder functionality is explored which measures bladder wall movements, which are compared to conventional bladder pressure measurements. An exploratory study was performed on rats, by placing an accelerometer on the bladder muscle and recording accelerations during voiding. The promising results of this preparatory study led to the development of a fully implantable system that can be used for long-term monitoring of Göttingen minipigs. The implant measures the mechanical acceleration of the bladder wall in four locations, the pressure between bladder muscle wall and the mucous membrane and the abdominal reference pressure. The sensors are placed on a flexible substrate that allows the measurement system to stretch with the bladder. The system uses a wireless telemetry link to configure the implant settings and sending all sensor data to a base station outside the body, using the $430 \mathrm{MHz}$ ISM band. With an idle current consumption of $10 \mathrm{uA}$ and $3.1 \mathrm{~mA}$ during sampling and wireless transmission of the data, a battery-powered autonomy of several weeks was 
achieved using a smart communication scheme. The telemetry system can communicate with a base station in the living quarters of the pigs at a distance of several meters. Several cadaveric tests were performed to validate the functionality of the system, followed by a long-term in vivo implantation, of which preliminary data is shown.

Acknowledgments: This work was supported by the European Research Council under the European Union's Seventh Framework Programme (FP7/2007-2013)/ERC grant agreement nº 340931.

Ethical Statements: The animal experiments in this work were evaluated and approved by the Animal Ethics Committee (project numbers 079/2015 and 172/2015) of the KU Leuven in compliance with all applicable national and international guidelines.

Declaration of interests: none.

\section{References}

[1] D. Irwin, Z. Kopp, B. Agatep, I. Milsom, P. Abrams, Worldwide prevalence estimates of lower urinary tract symptoms, overactive bladder, urinary incontinence and bladder outlet obstruction, BJU Int. 108 (2011) 1132-1139. doi:10.1111/j.1464-410X.2010.09993.x.

[2] W. Schfer, P. Abrams, L. Liao, A. Mattiasson, F. Pesce, A. Spangberg, A.M. Sterling, N.R. Zinner, P. Van Kerrebroeck, Good Urodynamic Practices: Uroflowmetry, filling cystometry, and pressureflow studies, Neurourol. Urodyn. 21 (2002) 261-274. doi:10.1002/nau.10066.

[3] D.M. Barrett, A.J. Wein, Flow evaluation and simultaneous external sphincter electromyography in clinical urodynamics., J Urol. 125 (1981) 538-541. doi:10.1016/S0022-5347(17)55098-3.

[4] J.I. Gillespie, The autonomous bladder: A view of the origin of bladder overactivity and sensory urge, BJU Int. 93 (2004) 478-483. doi:10.1111/j.1464-410X.2003.04667.x.

[5] M.J. Drake, A. Kanai, D.A. Bijos, Y. Ikeda, I. Zabbarova, B. Vahabi, C.H. Fry, The potential role of unregulated autonomous bladder micromotions in urinary storage and voiding dysfunction; overactive bladder and detrusor underactivity, BJU Int. 119 (2017) 22-29. doi:10.1111/bju.13598.

[6] T. Zagar, D. Krizaj, Validation of an acceierometer for determination of muscie beiiy radial displacement, Med. Biol. Eng. Comput. 43 (2005) 78-84.

[7] L. Brancato, T. Weydts, M.A. Soebadi, D. De Ridder, R. Puers, Submucosal Exploration of EMG and Physiological Parameters in the Bladder Wall, Proceedings. 1 (2017) 605. doi:10.3390/proceedings1040605.

[8] C.A. Maggi, P. Santicioli, A. Meli, The nonstop transvesical cystometrogram in urethaneanesthetized rats: A simple procedure for quantitative studies on the various phases of urinary bladder voiding cycle, J. Pharmacol. Methods. 15 (1986) 157-167. doi:10.1016/01605402(86)90064-1.

[9] P.A. McAnulty, A.D. Dayan, N.-C. Ganderup, K.L. Hastings, The minipig in biomedical research, 2012. doi:10.1201/b11356.

[10] P. Vodička, K. Smetana, B. Dvořánková, T. Emerick, Y.Z. Xu, J. Ourednik, V. Ourednik, J. Motlík, The miniature pig as an animal model in biomedical research, in: Ann. N. Y. Acad. Sci., 2005: pp. 161-171. doi:10.1196/annals.1334.015. 
[11] M.M. Swindle, A. Makin, A.J. Herron, F.J. Clubb, K.S. Frazier, Swine as Models in Biomedical Research and Toxicology Testing, Vet. Pathol. 49 (2012) 344-356. doi:10.1177/0300985811402846.

[12] S.J.A. Majerus, P.C. Fletter, E.K. Ferry, H. Zhu, K.J. Gustafson, M.S. Damaser, Suburothelial bladder contraction detection with implanted pressure sensor, PLoS One. 12 (2017) e0168375. doi:10.1371/journal.pone.0168375.

[13] M. Pinkawa, B. Asadpour, J. Siluschek, B. Gagel, M.D. Piroth, C. Demirel, M.J. Eble, Bladder extension variability during pelvic external beam radiotherapy with a full or empty bladder, Radiother. Oncol. 83 (2007) 163-167. doi:10.1016/j.radonc.2007.03.015.

[14] F. Merli, L. Bolomey, J.F. Zürcher, G. Corradini, E. Meurville, A.K. Skrivervik, Design, realization and measurements of a miniature antenna for implantable wireless communication systems, IEEE Trans. Antennas Propag. 59 (2011) 3544-3555. doi:10.1109/TAP.2011.2163763.

[15] F. Merli, L. Bolomey, F. Gorostidi, B. Fuchs, J.F. Zürcher, Y. Barrandon, E. Meurville, J.R. Mosig, A.K. Skrivervik, Example of data telemetry for biomedical applications: An in vivo experiment, IEEE Antennas Wirel. Propag. Lett. 11 (2012) 1650-1654. doi:10.1109/LAWP.2013.2238500.

[16] A. Kiourti, K.S. Nikita, Recent Advances in Implantable Antennas for Medical Telemetry [Education Column], IEEE Antennas Propag. Mag. 54 (2012) 190-199. doi:10.1109/MAP.2012.6387813.

[17] S. Gabriel, R.W. Lau, C. Gabriel, The dielectric properties of biological tissues: II. Measurements in the frequency range $10 \mathrm{~Hz}$ to $20 \mathrm{GHz}$, Phys. Med. Biol. 41 (1996) 2251-2269. doi:10.1088/00319155/41/11/002.

[18] Y.E. Mohammed, A.G. Saber, Estimation of E-Field inside Muscle Tissue at MICS and ISM Frequencies Using Analytic and Numerical Methods, J. Biomed. Eng. Technol. 2 (2014) 29-33. doi:10.12691/JBET-2-3-1.

[19] L. Brancato, R. Puers, Multi-layer embedded carbon fibres as highly compliant and stretchable interconnects, Flex. Print. Electron. 3 (2018). doi:10.1088/2058-8585/aaae75. 